REVISTA DE DERECHO ECONÓMICO

$$
\begin{array}{r}
\text { LOS ÓRGANOS DE LA } \\
\text { ADMINISTRACIÓN DEL } \\
\text { ESTADO COMO SUJETOS DE } \\
\text { LAS MULTAS APLICADAS POR } \\
\text { ELTRIBUNAL DE DEFENSA DE } \\
\text { LA LIBRE COMPETENCIA } \\
\text { WLLAM GARCIAMACHMAR }
\end{array}
$$




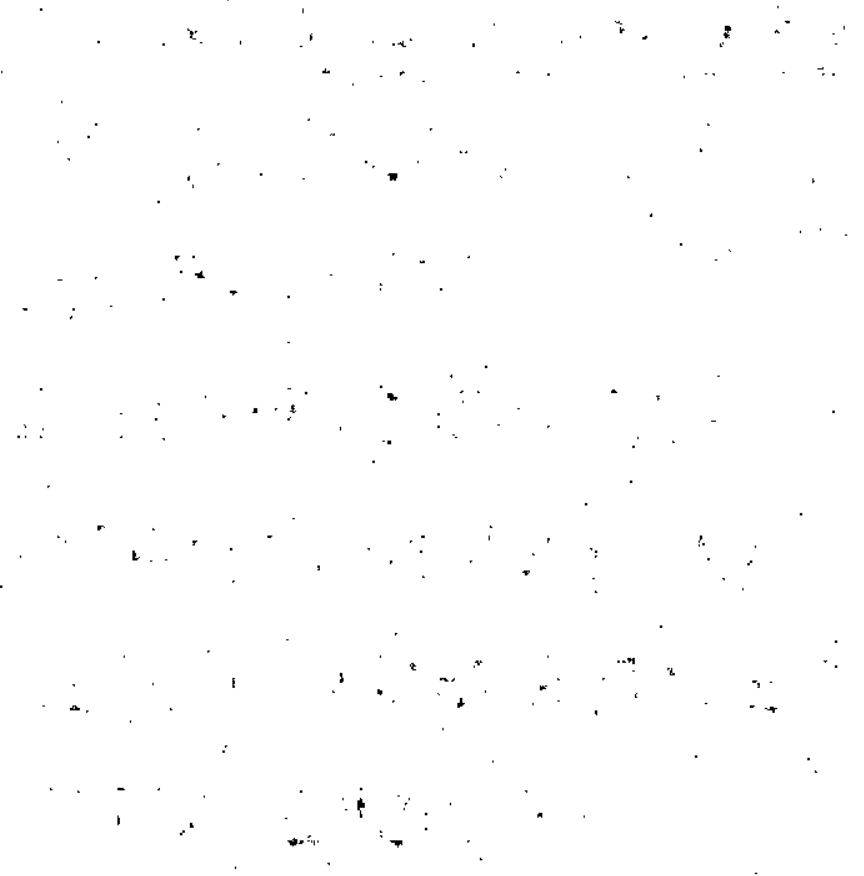




\title{
LOS ÓRGANOS DE LA ADMINISTRACIÓN DEL ESTADO COMO SUJETOS DE LAS MULTAS APLICADAS POR EL TRIBUNAL DE DEFENSA DE LA LIBRE COMPETENCIA
}

\author{
WILLIAM GARCÍA MACHMAR*
}

\section{RESUMEN}

El autor examina los problemas de la aplicación de multas por el Tribunal de Defensa de la Libre Competencia, en cuanto pueden afectar a los órganos de la Administración del Estado y propone una posible interpretación a la luz del derecho administrativo.

Palabras clave: derecho de la competencia, multa, potestad administrativa, presunción de legalidad, contencioso-administrativo, órganos de la Administración del Estado.

\section{INTRODUCCIÓN'}

El objeto del trabajo se centra en el análisis de la multa, como instrumento principal de la intervención pública en la competencia.

El presente trabajo pretende entregar una propuesta de interpretación para la aplicación de multas por parte del Tribunal de Defensa de la Libre Competencia (TDLC), particularmente en su imposición a los órganos de la Administración del Estado.

Para ello se emplearán los conceptos propios del derecho público administrativo, haciendo cierto contraste con la doctrina dominante en nuestro país en la materia. Se trata, por tanto, de aplicar el sistema conceptual de

* Licenciado en Ciencias Jurídicas y Sociales, Magíster en Derecho, mención Derecho Público, U. de Chile y Master of Laws, New York University. Agradezco a Anita Martínez Chamorro por sus comentarios a la redacción final y su ayuda para corregirla.Todos los errores son de responsabilidad del autor.

Diciembre de 2013.

I Normas citadas: $L D C=D F L N^{\circ}$, de 2005, Ministerio de Economía, Fomento y Reconstrucción; LOCBGAE = Ley Orgánica Constitucional de Bases Generales de la Administración del Estado, DFL $N^{\circ}$ I de 2001/19.653, Ministerio Secretaría General de la Presidencia de la República; LBPA $=$ Ley $N^{\circ} 19.880$ de bases de los procedimientos administrativos. 
esta rama del derecho como un modo de darle coherencia a la realidad nacional.

El trabajo analizará la doctrina dominante en Chile en cuanto al destinatario de las normas de defensa de la competencia. Luego, se abordarán los problemas relativos a la aplicación de multas a órganos de la Administración del Estado y se analizará críticamente la directiva de la FNE sobre la materia. A continuación, se propondrá una interpretación de los preceptos legales y una tipología de la jurisprudencia actual del TDLC. Finalmente, se entregará una breve noticia de la situación en los ordenamientos norteamericano y español.

\section{EL DESTINATARIO DE LAS NORMAS DE DEFENSA DE LA COMPETENCIA: ELTITULAR DE LA LIBERTAD ECONÓMICA}

El derecho de la competencia surge de la necesidad de limitar la libertad privada, mediante normas de orden público "económico", con el fin de lograr una economía "libremente" competitiva. Por eso, el derecho de la competencia cobra eficacia mediante técnicas de limitación de la libertad: multas, órdenes y prohibiciones, como las que enumera de hecho el artículo 26 LDC 211 . Dicho de otro modo, es una forma de intervención pública en la economía, con ciertas características especiales que ya veremos.

Como consecuencia de lo anterior, resulta lógico que el destinatario natural de las normas que regulan son los particulares, es decir, los titulares del derecho a la libertad (artículo $1^{\circ}$, inciso $1^{\circ}$ en relación con los artícu$\operatorname{los} 19 \mathrm{~N}^{\circ} 7^{\circ}$ y $\mathrm{N}^{\circ} 21$ de la Constitución). El hecho de que es el particular y no los órganos de la Administración el titular del derecho es obvio para la doctrina constitucional? .

2 Sobre la titularidad privado de este derecho, la doctrina constitucional es unánime. Por todos, v. FERMANDOIS VÖHRINGER, Arturo, "Derecho Constitucional Económico", T. I, $2^{\text {a }}$ ed., Santiago, Ed. Universidad Católica, 2006, pp. 219-220, con referencias "filosóficas, de historia fidedigna y de texto constitucional y legal". Vid. también GARCÍA VITORIA. Ignacio, "La libertad de empresa: ¿Un terrible derecho?", Madrid, Centro de Estudios Politicos y Constitucionales, 2008, pp. III-138, en que enfatiza la conexión entre el derecho y la esfera de autorrealización del individuo, esto es. persona natural. 
Ahora bien, la ley no limita expresamente la naturaleza jurídica del sujeto que puede ser el infractor del orden competitivo (pues se limita a decir que será "el que" realice ciertas conductas). Con fundamento en esa cláusula amplia, la doctrina y la jurisprudencia dominantes en Chile han entendido que el destinatario de la norma es cualquier "ente" jurídico, cualquiera sea su calidad ${ }^{3}$.

El único criterio es determinar a quién se le imputa la infracción, de acuerdo con las reglas generales del artículo $3^{\circ}$ sobre el ámbito subjetivo del ilícito anticompetitivo ${ }^{4}$. Las condiciones para imputar responsabilidad pueden sintetizarse de la siguiente manera: "se impone la sanción a quien, conociendo o debiendo conocer que una conducta está prohibida y castigada con una sanción, y siendo libre para llevarla a cabo o no, la realiza, violando la norma sancionadora"5.

EITDLC ha sido enfático en afirmar que su competencia es amplia, desde el punto de vista subjetivo, declarando que "los artículos $3^{\circ}$ y $18^{\circ} \mathrm{N}^{\circ} 1$ del Decreto Ley $N^{0} 211$ atribuyen competencia a este Tribunal para conocer y resolver respecto de cualquier situación que pudiere constituir una infracción a la libre competencia, esto es, cualquier hecho, acto o convención que impida, restrinja o entorpezca la libre competencia, o tienda a producir dichos efectos, sin que se prevean excepciones o limitaciones a ella, derivadas de la calidad o naturaleza jurídica del sujeto activo que pudiera haber cometido tales infracciones".6.

En el mismo sentido se ha pronunciado la doctrina dominante. Así, Valdés Prieto afirma que la fórmula del artículo $3^{\circ} \mathrm{DL} 211$ " comprende toda persona natural o jurídica, de derecho privado o público, sea que obre personalmente, a través de órganos, mandatarios o agentes oficiosos, ya sea que se trata de un simple particular, de una persona pública no comportándose como autoridad, sino como competidor de una autoridad privada o de una autoridad pública en ejercicio de potestades

3 V. infra nota 9

4 VALDÉS PRIETO, Domingo, "Libre Competencia y Monopolio", Santiago, Ed. Jurídica de Chile, 2006, pp. 250 y ss.

5 HUERGO LORA, Alejandro, "Las sanciones administrativas", Madrid, lustel, 2007, p. 396

6 Sentencia $N^{\circ} 100$, de 21 de julio de 2010 , sobre considerando $6^{\circ}$. 
normativas infralegales". Por su parte, Nehme Zalaquett afirma que "los órganos del Estado, todos ellos, se encuentran sujetos en la dictación de actos administrativos a las normas de defensa de la competencia"8.

Sin embargo, esta opinión, dominante en la doctrina y la jurisprudencia, debe ser analizada con fuertes reservas. A nuestro juicio no es obvio que ef TDLC tenga una potestad para revisar lo actuado por un órgano de la Administración del Estado. Existen razones, que examinaremos a continuación, que apuntan en dirección contraria a la doctrina mayoritaria.

\section{PROBLEMAS QUE SURGEN AL APLICAR MULTAS A LOS ÓRGANOS DE LA ADMINISTRACIÓN DEL ESTADO}

Ante todo, no basta con afirmar la primacía constitucional y citar las normas de la Constitución -como acabamos de ver que lo hace la doctrina dominante- para concluir que, por ese solo hecho, la competencia viene exigida perentoriamente por la Constitución, respecto de todo sujeto?.

La Constitución, si bien garantiza indudablemente ciertos derechos de libertad en materia económica, ha entregado las decisiones esenciales al legislador (artículo $63 \mathrm{~N}^{\circ} 20$ ). Luego es en la ley que debemos buscar la definición de los deberes recíprocos entre los partícipes de un mercado. Además, en la misma ley están definidas potestades públicas que se confían a los órganos de la Administración del Estado.

Trataremos, en primer lugar, los problemas de diseño procesal que dificultan extender la aplicación de multas a los órganos de la Administración para luego, en segundo lugar, abordar el carácter central que tiene el artículo $4^{\circ}$ del $D L 21$ I como cobertura a la actuación pública. Por último, se analiza críticamente la directiva de la FNE sobre la materia.

7 VALDÉS PRIETO, Domingo, Op. cit., p. 251.

8 NEHME ZALAQUETT, Nicole, "Aplicación de las normas de defensa de la competencia a los órganos de la Administración del Estado", en Tribunal de Defensa de la Libre Competencia (ed.) La Libre Competencia en el Chile del Bicentenario, Santiago, Thomson Reuters, pp. 328-329.

9 NEMME ZALAQUETT, Nicole, Op. cit., pp. 320-322. 


\section{Problemas de procedimiento}

EI TDLC no es un tribunal contencioso-administrativo. Más bien, se trata de un tribunal especial que puede llegar a conocer de acciones dirigidas contra órganos de la Administración del Estado, pero que por regla general conoce de requerimientos contra privados. Así está configurado el procedimiento. Esto significa que varias de las instituciones del proceso no están diseñadas para la especialidad de la función administrativa.

Por de pronto, el procedimiento contempla la posibilidad de conciliación (artículo 22), institución que sólo en casos excepcionales podría aplicarse a los órganos de la Administración del Estado, los que ejercen potestades públicas (artículo $6^{\circ}$ LOCBGAE), las que son, evidentemente, indisponibles para su titular ${ }^{10}$.

La misma norma establece los medios de prueba, el procedimiento y el estándar de convicción. Sin embargo, es necesario recordar que, tratándose de los órganos de la Administración del Estado, la carga probatoria se invierte, pues sus actos gozan de presunción de legalidad (artículo $3^{\circ}$ LBPA)", de modo que el estándar de la "sana crítica" sólo puede aplicarse en la medida que el particular haya logrado derribar la presunción de legalidad. En consecuencia, la declaración de ilegalidad es un supuesto necesario para acoger la acción.

Además, el artículo 25 otorga alTDLC una amplia facultad para ordenar medidas cautelares. Al respecto, cabe recordar que, en principio, los actos administrativos gozan de ejecutoriedad, de modo que, aunque sean impugnados, obligan y deben cumplirse tan pronto como sean puestos en conocimiento de sus destinatarios ${ }^{12}$.

10 Por todos, véase SANTAMARÍA PASTOR, Juan Alfonso. "Principios Generales de Derecho Administrativo", T. I, Madrid, lustel, 2006, p. 424.

11 Se trata de una presunción toda vez "que permite al acto desplegar todos sus posibles efectos en tanto no se demuestre su invalidez y que traslada. en consecuencia, al particular la carga de impugnarlo, en la vía administrativa o contencioso-administrativa, según proceda, si quiere obtener su anulación y frenar su eficacia". (García de Enterría, Eduardo y Fernández, Tomás-Ramón, "Curso de Derecho Administrativo", T. $1,12^{a}$ ed., Madrid-Buenos Aires, CivitasLa Ley, 2004, p. 585).

12 Artículos $3^{\circ}$, inciso final, 50,51 y 52 LBPA. 
Si bien es cierto que la orden de suspensión dictada por un tribunal interrumpe la eficacia del acto administrativo (artículo $3^{\circ}$ inciso final LBPA), el citado artículo del LDC 21 I no se sitúa en la hipótesis que la suspensión de un acto de la autoridad pública pueda poner en grave riesgo el interés público e, incluso, los derechos de terceros legítimamente interesados en su eficacia. Entonces, la ponderación entre los intereses en juego será una tarea que estará sólo en manos del juez, con el evidente riesgo de arbitrariedad judicial, pues no hay estándares legales a los que acogerse.

Finalmente, la ley no arbitra ningún medio especial para hacer cumplir coactivamente las resoluciones judiciales frente a un órgano del Estado. Aunque el artículo 29 se remite, supletoriamente, al Código de Procedimiento Civil, se limita a los Libros I y II, los que no prevén ninguna hipótesis especial tratándose de la ejecución contra la Administración. El único procedimiento en que sí se tratan estos medios es el juicio de hacienda, el que no está comprendido en la remisión ${ }^{13}$.

De lo anterior se extrae que el procedimiento regulado por la LDC está pensado de forma que el sujeto pasivo de la multa será un privado, pero no se pone en la hipótesis que el sujeto involucrado es un órgano de la Administración del Estado.

\section{Carácter central del artículo $4^{\circ}$ de la Ley de Defensa de la Competencia}

Es necesario resaltar la importancia que tiene la ley como ámbito de referencia inmediato de la actuación administrativa.Tratándose de órganos de la Administración del Estado debemos suponer que actúan siempre con "autorización legal" previa (artículo $2^{\circ}$ LOCBGAE). Luego, en principio, toda intervención de estos órganos está amparada en la excepción del artículo $4^{\circ}$ LDC, que prohíbe los monopolios "salvo que la ley lo autorice".

"ARTICULO 4:- ND PDDRÁN DTORGARSE CONCESIONES, AUTORIZACIONES, NI ACTOS QUE IMPLIQUEN CONCEDER MONOPDLIOS PARA EL EJERCICID DE ACTMIDADES ECONÓMICAS, SALVO QUE LA LEY LD AUTORIEE":

Dicho de otro modo, la defensa de la competencia reconoce como excepción aquellas actuaciones realizadas con "autorización" legal; y toda actuación administrativa se presume que cuenta con ella.

I3 NEHME ZALAQUETT, Nicole, Op. cit., pp. 332-333. 
Entonces, puede sostenerse que, desde el punto de vista de los fines de la regulación, el régimen de derecho público, gobernado por el principio de legalidad, se exceptúa -0 , lo que es lo mismo, "es exorbitante"- respecto del régimen de defensa de la competencia. Sobre la personalidad de la Administración y sus privilegios exorbitantes, que acompañan el ejercicio de toda potestad, existen excelentes trabajos ${ }^{14}$, que no corresponde reproducir acá, más aún si se tiene en cuenta el expreso reconocimiento de estos privilegios exorbitantes en la LBPA. En efecto, todos los actos administrativos, incluso los que el TDLC pueda revisar en ejercicio de su competencia, gozan de presunción de legalidad (artículo $3^{\circ}$ inciso final LBPA $^{15}$ ) y, por lo tanto, no pueden enjuiciarse del mismo modo que las actuaciones privadas, que no tienen este privilegio. En consecuencia, para aplicar una multa a un órgano de la Administración del Estado, será necesario, entonces, como supuesto lógico previo, vencer esta presunción y que el Tribunal declare la ilegalidad de la actuación administrativa.

A mayor abundamiento, el texto del artículo $3^{\circ}$ LDC, ya en su hipótesis genérica del inciso primero, así como en los literales del mismo artículo, emplean un lenguaje que está dirigido a los particulares ("hecho, acto o convención”," competidores”, “agente económico”, etc.). Sólo por analogía y mediante una interpretación extensiva pueden aplicarse estas hipótesis a los órganos de la Administración del Estado.

Los argumentos que utiliza Valdés - el empleo de la voz "el que", los números 21 y 22 del artículo 19 de la Constitución, y la jurisprudencia

14 En Chile, FERRADA BÓRQUEZ، Juan Carlos. "Las potestades y privilegios de la Administración pública en el régimen administrativo chileno", en Revista de Derecho (Valdivia) [online]. 2007. vol. 20, n. 2, pp. 69-94. En España véase GARCÍA DE ENTERRÍA Eduardo y FERNÁNDEZ, Tomás-Ramón, Op. cit., T. I, pp. 29-65. Incluso en Estados Unidos se llega a consecuencias prácticas que son del todo similares a las de nuestro derecho después del fallo Chevron. BREYER, Stephen; STEWART, Richard B.; SUNSTEIN, Cass; VERMEULE, Adrian y HERZ, Michael E., "Administrative Law and Regulatory Policy", $7^{\mathrm{a}}$ ed., New York, Wolters Klower, PP. 288: "APA $\S 706$ says that 'the reviewing court shall decide all relevant questions of law' (emphasis added). That provision is consistent with the old saw from Marbury v. Madison that it is 'emphatically the province and duty of the judicial department to say what the law is.' Chevron seems in conflict with those familiar and basic propositions, it can thus be seen as a kind of counter-Marbury for the administrative state".

15 "Los actos administrativos gozan de una presunción de legalidad, de imperio y exigibilidad frente a sus destinatarios, desde su entrada en vigencia...". 
previa de la Comisión Resolutival6 - no se hacen cargo de esta incoherencia recién señalada.

En un reciente trabajo, Nehme Zalaquett identifica correctamente que el TDLC debe dilucidar, ante todo, cuál es el nivel de sujeción a la ley de determinado acto administrativo. Dependiendo de este nivel de sujeción podremos determinar cuál es la capacidad que tiene el TDLC para intervenir en la actuación administrativa. Esa aproximación, como se ha dicho, es correcta. Sin embargo, la conclusión a la que llega la autora es completamente ajena al sistema de derecho público y al control judicial de los actos administrativos, según el cual el control de los actos administrativos depende directamente de si la ley ha regulado exhaustivamente su contenido o no, lo que es lo mismo, según si son reglados o discrecionales. Esta distinción entre actos reglados y discrecionales es central en el derecho administrativo, porque afecta el nivel de control sobre la actuación administrativa.

Para Nehme Zalaquett, el poder de revisión del TDLC es más intenso tratándose de los actos administrativos discrecionales $y$, en cambio, más débil tratándose de los actos reglados. Esta afirmación se extrae de dos pasajes del referido artículo en que se sostiene, por una parte, que la Corte Suprema actuó correctamente revocando una sentencia del TDLC que ordenaba a la Junta de Aeronáutica Civil modificar el sistema de adjudicación de las frecuencias de vuelo entre Santiago y Lima 17; y, por otra parte, que también la Corte Suprema tenía la potestad de limitar la facultad discrecional de la Subsecretaría de Telecomunicaciones para licitar las frecuencias de telefonía móvil digital ${ }^{18}$.

La conclusión de la autora está en agudo contraste con la doctrina tradicional del derecho administrativo, según la cual:

"SI LA LEY RENUNCIA A REGULAR LA DISCRECIONALIDAD Y ÉSTA CONIISTE MÁS BIEN EN QUE LA LEY SE REMITE A LA APRECIACIÓN SUBJETIVA DE LOS AGENTES DE LA ADMINISTRACIÓN, NO HAY MODD DE ARTICULAR UN CONTROL DE LEGALIDAD DE SU EJERCICIO; SÓLO PODRÁ VALORARSE SU OPORTUNIDAD, PERO ESTO SERÁ PROPIO DE UN JUICIO POLITICO (PODRÁ SER POR ELLO

16 VALDÉS PRIETO, Domingo. Op. cit., pp. 252-254.

17 NEHME ZALAQUETT, Nicole. Op. cit., pp. 336-338.

18 Ibíd., pp. 342-344 
CONTRQLADO POR UN ORGANO POLITICD...), NO SERÁ EN NINGÚN CASO ARTICULABLE EN UN JUICO DE DERECHO, QUE ES LO PROPIO DE LOS TRIBUNALES CONTENCIOSD-ADMINISTRATINDS" $"$ ?

Dicho de otro modo, cuando la ley ha programado completamente la actividad administrativa, el control debe tener por objeto examinar su sujeción a la ley, pues es esta la que actúa como estándar de referencia inmediato. Pero cuando la ley ha entregado un ámbito de decisión autónomo a la Administración, no cabe control jurídico. Ello no vulnera o ignora el principio de legalidad, sino que es justamente una aplicación de este. Si fuera posible controlar jurídicamente ese espacio de discrecionalidad, entonces esta por definición deja de existir ${ }^{20}$.

A modo de conclusión, el artículo $4^{\circ} \mathrm{DL} 21$ I establece que el derecho de la competencia no se aplica ahí donde la ley ha establecido una excepción. Sabemos también que toda actuación administrativa debe estar habilitada por ley. Por lo tanto, la idea que pretendemos sugerir es que, en términos generales, la actuación administrativa no se sujeta al derecho de la competencia del mismo modo que la actuación privada.

\section{Análisis crítico de la directiva "Sector Público y Libre Competencia" de la FNE}

Antes de proceder a la propuesta interpretativa de este artículo, cabe hacer referencia a un hecho de reciente acontecimiento. En junio de 2012 la Fiscalía Nacional Económica (FNE) publicó una directiva en que intenta orientar la actuación administrativa en materia económica. Su propósito primordial es entregar un método para examinar el impacto de las actuaciones del "sector público" sobre la competencia en los mercados.

En el documento se identifica correctamente que:

"LLJOS ÓRGANOS DE LA ADMINISTRACIÓN DEL ESTADO ('OAE') U ORGANISMOS DEL SECTDR PÚBLICD EN EL EJERCICIO DE SUS FACULTADES Y EN CUMPLIMIENTO DE LOS FINES PARA LOS QUE FUERON CREADOS INTERACTÚAN CON LOS MERCADOS. ESTAS INTERACCIONES PUEDEN ADDPTAR DIVERSAS FORMAS -ACTUANDD COMO AGENTE ECONÓMICO (POR EJEMPLO,

19 GARCÍA DE ENTERRÍA, Eduardo y FERNÁNDEZ, Tomás-Ramón, Op. cit. T. I. pág. 464. Véase también MAURER, Hartmut. "Derecho Administrativo. Parte General", Marcial Pons, Madrid, 2011, pp. 166-167.

20 Sobre el concepto de discrecionalidad, cómo se reduce y cuáles son las técnicas de control véase SAAVEDRA FERNÁNDEZ, Rubén. "Discrecionalidad Administrativa". Santiago, Abeledo Perrot, 2011. 
COMPRANDO BIENES O SERVICIOS PARA SU OPERACIÓN), EN LA EJECUCIÓN DE PLANES Y PROGRAMAS PROPIOS DE SUS FUNCIONES, O EN EL EJERCICIO DE SU POTESTAD NORMATIVAY REGULATORIA- LAS CUALES DAN LUGAR A UNA CONSTANTEY ESENCIAL INFLUENCIA SOBRE LAS DECISIONES DE OTROS AGENTES ECONOMICOS; POR LO ANTERIOR, LAS ACTUACIONES DE LOS OAE GENERALMENTE TIENEN EFECTOS SOBRE LA COMPETENCIA"21.

En efecto, la distinción que ofrece la FNE entre la gestión económica, la prestación de servicios y la regulación del mercado es importante y de ella se derivan consecuencias jurídicas para el derecho de la competencia -como desarrollaré más adelante. Sin embargo, la FNE no ahonda en esa distinción y afirma a continuación que:

"SOBRE ESTE PUNTO, CABE TENER PRESENTE QUE LA LEGISLACIÓN DE LIBRE COMPETENCIA EN NUESTRO PAIS NO EXIME A ORGANO ALGUNO -SEA UNA ENTIDAD PÚBLICA O PRIVADA, Y CUALQUIERA SEA SU ORGANIZACIÓN O ESTRUCTURA SOCIETARIA- DEL CUMPLIMIENTO DEL DL 211, NI ESTABLECE EXCEPCIDNES A MERCADOS DETERMINADOS. DE MANERA QUE EN TODOS LOS MERCADOS DEBE CUMPLIRSE CON LA NORMATIVA DE LIBRE COMPETENCIA POR TODOTIPD DE ENTIOAD O NOMIOUO"22

Por lo tanto, aplica sin ningún tipo de distinción el estándar del artículo $3^{\circ}$ de la LDC 211 . Sin embargo, el artículo $4^{\circ}$ de dicha ley admite que existan políticas públicas que dispongan excepciones o estén en contradicción con el derecho de la competencia:

\footnotetext{
"EN LA MEDIDA QUE ESTAS DISPOSICIONES ESTÉN FUNDADAS EN UN MANDATO LEGAL EXPRESO Y ESPECIFCO QUE SE SDBREPONGA A LA NORMATIVA DE LIBRE COMPETENCIA, Y QUE LA ACTUACIÓN DE LOS OAE SE CIÑA ESTRICTAMENTE A LO DISPUESTO EN LA LEY, LA FNE ENTIENDE QUE TAL ACTUACIÓN NO SERIÁ OBJETO DE REPROCHE COMO UICITO ANTICOMPETTIVO"23.
}

Entonces, a juicio de la Fiscalía, la clave está en distinguir entre el ejercicio de potestades regladas y discrecionale ${ }^{24}$. Sin embargo, en una reinterpretación del sentido de las potestades discrecionales, sostiene que es precisamente tratándose de ellas que aparece con más fuerza el control de ella misma, la Fiscalía, y elTDLC. En efecto, se sostiene en el documento que:

21 Fiscalía Nacional Económica, Sector Público y Libre Competencia. Evaluando sus actuaciones desde la perspectiva de la competencia. Junio de 2012, p. 4. Destacado en el original, se han omitido las notas al pie.

22 tbid., pp. 4-5.

23 ibíd., p. 5.

24 ibid., pp. 5, 8-9. 
"LAS ACTUACIDNES DE LOS ORGANISMOS PÚBLICDS QUE ESTAN EXPRESAMENTE NORMADAS PDR LEY, NO CONSTITUYEN UN ILICITO ANTICOMPETITIVO, EN LA MEDIDA QUEESTÁN CDMPLETA E INTEGRAMENTE AMPARADAS POR EL PRINCIPIO DE LEGALIDAD.

LAS ACTUACIDNES REALIZADAS EN EJERCICID DE LA PDTESTAD DISCRECIDNAL DE LOS DAE Sf ESTÁN SUJETAS A EVALUACIÓN Y SANCIÓN DE PARTE DE LAS AUTORIDADES DE COMPETENCIA EN LA MEDIDA QUE TRANSGREDAN EL DL 211, SIN QUE SE APLIQUE NINGUNA EXCEPCION O EXENCIÓN"25.

De esta manera, la Fiscalía declara que ampliará sus facultades de control precisamente ahí donde la ley especial ha confiado a cada uno de los órganos administrativos la posibilidad de definir, sin un ámbito de referencia normativo, una política pública. Por el contrario, la directiva propone un método bien específico para ejercer esas potestades "discrecionales" -que ya dejarían de serlo- conforme una especie de sistema de evaluación de impacto competitivo ${ }^{26}$.

\section{ELTRIBUNAL DE DEFENSA DE LA LIBRE COMPETENCIA SÓLO EXCEPCIONALMENTE PUEDE APLICAR MULTAS A UN ÓRGANO DE LA ADMINISTRACIÓN DEL ESTADO}

Las contradicciones anteriormente identificadas obligan a reinterpretar la jurisprudencia del Tribunal, pues su doctrina constante en la materia es que se ha considerado competente para controlar la actuación de los órganos de la Administración del Estado. Por lo tanto, acá no cuestionaré esta competencia, que es un hecho, sino que me limitaré a examinar cómo la presencia de un órgano administrativo altera su respuesta represiva, es decir, la aplicación de multas.

La propuesta que acá se entrega parte de la base de distinguir los varios ámbitos en que actúa la Administración, para lo cual propongo una clasificación de los casos resueltos por elTDLC en que el sujeto pasivo ha sido un órgano de la Administración del Estado.

De este modo, podemos clasificar los casos, según los tipos de órganos involucrados, del siguiente modo, por una parte, aquellos que afectan a

25 lbíd., p. 12. Destacado en el original, se han omitido los gráficos.

26 lbid., pp. 14-23. 
las empresas del Estado y, por otra, aquellos que afectan a órganos que ejercen potestades públicas (artículo $6^{\circ}$ LOCBGAE).

\section{El Estado Empresario se rige, en general, por la Ley de Defensa de la Competencia}

Toda vez que las empresas del Estado y las sociedades en que éste tenga participación, actúan en general bajo las reglas del derecho común ${ }^{27}$ (art. $19 \mathrm{~N}^{\circ} 21 \mathrm{CPR}$ ), entonces, pueden perfectamente cometer ilícitos anticompetitivos, en los mismos términos que las empresas privadas ${ }^{28}$. Esto se fundamenta en el hecho que se trata de entes que, en todo rigor, compiten en un mercado. No es un obstáculo la forma que adopte la empresa, sea una empresa del Estado creada por ley o una sociedad en que el Estado tenga participación conforme a las reglas del derecho privado.

Con todo, es perfectamente posible que una ley de quórum calificado establezca reglas especiales que las exima de este régimen competitivo.

Precisamente en la sentencia $N^{\circ} 70$, de 02.07.2008, el TDLC estimó que los precios privilegiados que cobraba ENAMI se debían a su actividad de fomento a la minería, autorizada por ley, y por lo tanto no podían evaluarse como conductas anticompetitivas. En sus considerandos $22^{\circ}$ y $31^{\circ}$, argumentó que:

"ESTE TRIBUNAL CONSIDERA IMPOSBBLE QUE DICHA EMPRESA ABUSE DE LA POSICIÓN DE DOMINID QUE SOPDRTA POR EL SOLO CUMPLIMIENTO DE SU FUNCIÓN. QUE CONSISTE EN SUBSIDIARA LOS PEQUEÑOSY MEDIANOS MINEROS, EN EL SENTIDO DE OFRECER CONDICIONES DE COMPRA MÁS VENTAJDSAS QUE LAS QUE SE OBSERVARIAN EN UN MERCADO COMPETTTVD... EL ROL DE FDMENTO DE ENAMI, Y EN PARTICULAR SU DBLIGACIÓN DE PDSIBILITAR EL ACCESO A MERCADOS A LA PEQUEÑA Y MEDIANA MINERIÁ NO MPLICA EN NINGÚN CASO QUE DICHA EMPRESA ND PUEDA DIFERENCIAR ENTRE MINERALES DE DISTINTA CALIDAD. LO ANTERIOR, CDNSIDERANDD LA CAPACIDAD DE PRDCESAMIENTO LIMITADA DE LA DEMANDADA Y LA RELACIÓN EXPUESTA ENTRE LDS COSTOS REALES DE PRDCESAMIENTO DEL MINERAL Y LA LEY DEL MISMD"29.

27 PANTOJA BAUZÁ, Rolando, "La organización administrativa del Estado", Santiago, Ed. jurídica de Chile, 2004, pp. 178 y ss.

28 Véase por ejemplo, sentencia $N^{0} 76$, de 14.10.2008, confirmada por la CS, 13.01.2009, que acoge parcialmente requerimiento en contra de EFE y, sentencia $N^{\circ} 64$, de 18.04.2008, que rechaza demanda interpuesta contra ENAP.

29 Los mismos razonamientos se confirman en la sentencia de la Corte Suprema, 10.11.2008, considerando $7^{\circ}$. 


\section{Los órganos de la Administración del Estado que ejercen potestades públicas están, generalmente, exentos de la aplicación de multas por el TDLC}

Respecto del segundo grupo de casos, esto es, los órganos del Estado que ejercen potestades públicas (artículo $6^{\circ}$ LOCBGAE), podemos decir que son radicalmente distintos ${ }^{30}$. Puede decirse, incluso, que el solo hecho de que ejerzan potestades públicas los excluye del régimen competitivo $y$, entonces, respecto de ellos no podría aplicarse la LDC.

Con todo, sobre este punto puede establecerse una segunda distinción, según si el caso afecta la contratación pública o no.

a. El principio de libre concurrencia en la contratación pública faculta la intervención del Tribunal de Defensa de la Libre Competencia

En efecto, existe un grupo importante de casos en que el Tribunal ha debido pronunciarse sobre la licitud, desde el punto de vista de la competencia, acerca del procedimiento o sobre cláusulas introducidas en las bases de licitación ${ }^{3 !}$.

¿Cómo se explica en este caso la extensión de la competencia del TDLC sobre este tipo de casos? La interpretación que propongo acá es que, conforme al artículo $9^{\circ}$ LOCBGAE, la etapa de formación del contrato, en la medida que se sujete al procedimiento de licitación, que es la regla general, se debe regir por el principio de "libre concurrencia", Es decir, la ley convoca expresamente el régimen de defensa de la competencia.

"ARTICULO 9".- LOS CONTRATOS ADMINISTRATIVOS SE CELEBRARAN PREVIA PROPUESTA PÚBLICA. EN CONFORMIDAD A LA LEY.

EL PROCEDIMIENTO CONCURSAL SE REGIRA POR LOS PRINCIPIOS DE LIBRE CDNCURRENCIA DE LOS OFERENTES AL LLAMADO ADMINISTRATIVOY DE IGUALDAD ANTE LAS BASES QUE RIGEN EL CONTRATO.

LA LICITACIÓN PRIVADA PROCEDERÁ, EN SU CASO, PREVIA RESOLUCIÓN FUNDADA QUE ASÍ LO DISPONGA, SALVO QUE POR LA NATURALEZA DE LA NEGDCIACIÓN CORRESPONDA ACUDIR AL TRATO DIRECTO".

30 PANTOJA BAUZÁ, Rolando, Op. cit., pp. 229 y ss.

31 Sentencias $N^{\circ} \mid 14,92,91,89,81,77,44,37,34,2014,13,11$ y 4 . 
Cabe anotar que no son estos casos de contratación privada de la Administración, sino contratación que, rigiéndose por las reglas del derecho público, es decir, en que se ejercen potestades, se somete al principio de la libre concurrencia.

Esta idea es reconocida por la doctrina administrativa. En efecto, para Moraga Klenner, el artículo $9^{\circ}$ LOCBGAE exige "que la Administración no ponga más restricciones o limitaciones que las expresamente dispuestas en la ley, entre las cuales se destaca el marco legal de defensa de la competencia"32.

Es necesario precisar que en estos casos no es el Estado el que compite con actores privados, sino que este crea las condiciones para que los particulares compitan entre sí. La interpretación armónica de la LOCBGAE y la LDC nos lleva a concluir que para el legislador la Administración debe someterse a los estándares del TDLC cuando convoque la colaboración privada para el cumplimiento de sus tareas.

A diferencia del control que ejerce en esta materia el Tribunal de la Contratación Pública, acá estamos hablando de un control de fondo, es decir, del carácter competitivo o no del contrato considerado en sí mismo; no de su procedimiento.

La doctrina del TDLC ha sido sintetizada por Nehme Zalaquett del siguiente modo: no es procedente la incorporación de disposiciones en que los oferentes renuncien al derecho a reclamo o acción jurisdiccional; no pueden incorporarse exigencias que resulten en discriminaciones a favor de determinados agentes económicos; la evaluación de las ofertas no debe conferir amplia discrecionalidad al licitante por aumentar la incertidumbre y el costo del proceso; los criterios de evaluación deben contener una escala, confeccionada de acuerdo a distintos niveles de cumplimiento del criterio y no sólo un puntaje máximo; el licitante está facultado para desestimar ofertas o declarar desierta la licitación, siempre que posteriormente la adjudicación del servicio se efectúe mediante una nueva licitación; las licitaciones basadas únicamente en ofertas monetarias cuando los bienes a licitar son limitados, favorecen a la empresa

32 MORAGA KLENNER, Claudio, "Contratación administrativa", Santiago, Ed. Jurídica de Chile. 2007, p. 108. 
dominante; los bienes no deben ser asignados de modo indefinido, a menos de que existan razones que lo justifiquen, la exigencia de contar el proponente con determinados contratos vigentes es un requisito constitutivo de una barrera de entrada; y los procesos licitatorios deben contemplar plazos necesarios para posibilitar la entrega de ofertas serias por parte de los oferentes ${ }^{33}$.

En cualquier caso, el Tribunal ha sido deferente con la Administración. Del total de catorce $\operatorname{casos}^{34}$, ha rechazado nueve y acogido cinco. De estos últimos, en todos ellos ha impartido órdenes a los órganos de la Administración para que dejen sin efecto los procedimientos viciados y cambien sus prácticas futuras, sin aplicar multa ${ }^{35}$, salvo en un solo caso. En esa sentencia ( $N^{\circ}$ 77, de 04.1 1.2008), se aplicó a la Municipalidad de Curicó una multa de 5 UTA. El Tribunal, a pesar de aplicar la sanción, tasó su monto de manera discreta pues estimó que su aplicación significaba un perjuicio económico a la población, adicional al hecho de haberse celebrado los contratos respectivos con infracción a la normativa de competencia (considerando $70^{\circ}$ ).

Ahora bien el problema que resta por dilucidar es la posibilidad que el TDLC aplique multas en aquellos casos en que los órganos administrativos ejercen puramente potestades públicas, fuera del contexto de la contratación.

\section{b. El ejercicio de potestades públicas impide la aplicación de multas a los órganos de la Administración del Estado}

Para terminar, analizaré la situación en que el TDLC controla el ejercicio de potestades públicas que, sin implicar la celebración de un contrato, inciden en los mercados.

El Tribunal ha afirmado categóricamente que está facultado para extenderse sobre estas materias en su sentencia $N^{\circ} 100$ de 21.07.201036:

33 NEHME ZALAQUETT, Nicole, Op. cit., pp. 340-342.

34 Sentencias $N^{\circ}$ s 114, IOS, 100, 92, 91,89,81,77, 67, 44, 37, 34, 20,14, 13,11 y 4.

35 Sentencias $N^{\circ}$ s $114,105,100,81,44,37$ y 34.

36 Se reitera en sus sentencias $N^{\circ} s \mid 14,121$ y 123. 
"DÉCIMO: QUE, ESTABLECIDO LO ANTERIOR, CORRESPONDE DETERMINAR SI EN ESTE CASO LAS CONDUCTAS DENUNCIADAS DEBEN SUJETARSE A LAS NORMAS DE DEFENSA DE LA LIBRE COMPETENCIA.AL RESPECTO, CABE TENER PRESENTE QUE LOS SERVICIOS PÜBLICOS QUE PRESTA EL ESTADO -YA SEA DIRECTAMENTE O POR MEDIO DE CONCESIONARIDS $-Y$ POR LOS CUALES SE DEBE PAGAR UN PRECIO -SEA POR PARTE DE LOS USUARIOS O BENEFICIARIOS DIRECTOS O POR EL ESTADO EN REPRESENTACIÓN DEL INTERÉS PÚBLICO QUE PUDIERA EXISTIR EN LA PROVISIÓN DE DICHOS SERVICIOS- CONSTITUYEN UN MERCADO ANÁLOGO A CUALQUIER OTRO. EN EL SENTIDO QUE EXISTEN EN ÉL OFERENTES Y DEMANDANTES QUE TRANSAN BIENES O SERVICIOS EN DETERMINADAS CANTIDADESY PRECIOS;

UNDÉCIMO: QUE POR CONSIGUIENTE, Y CONSIDERANDO QUE EL DECRETO LEY No 211 ES APLICABLE A TODA CLASE DE ACTIVIDADES ECONÓMICAS, SEA QUE CORRESPONDAN D NO A LA PRESTACIÓN DE SERVICIOS PÚBLICOS, Y SEA QUE LOS PRESTE EL ESTADO DIRECTAMENTE O MEDIANTE CONCESIONARIOS, LA FORMA COMO EL ESTADO DESIGNE AL PRDVEEDOR DE UN BIEN O SERVICIO, ESPECIALMENTE PORMEDIO DEL DISEÑO DEL SISTEMA DE LICITACIÓN, TENDRA EFECTOS EN EL MERCADO EN QUE ÉSTA INCIDE. DEAHI QUE LOS EFECTOS EN LA COMPETENCIA -O FALTA DE ELLA- CAUSADOS POR LA FORMA EN QUE EL ESTADO REALIZA ESTAS LICITACIONES, AFECTAN FINALMENTE EL BIENESTAR GENERAL;

OUOOÉCIMO: QUE EN CONSECUENCIA, EN OPINIÓN DE ESTOS SENTENCIADDRES, EL ESTADO

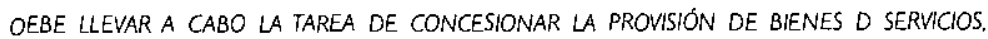
ASI COMO OESARROLLAR CUALQUIER FUNCIÓN QUE LE ENCOMIENDE EL ORDENAMIENTO JURIDICO Y QUE IMPLQUUE SU INTERVENCIÓN EN LDS MERCADOS, OBSERVANOO LAS NORMAS DE OEFENSA OE LA LIBRE COMPETENCIA, OE CUYA APLICACIÓN NINGUNA NORMA LO EXIME" (ÉNFASIS AGREGADO).

Es importante destacar que a pesar de la contundencia de la doctrina emanada de esta sentencia, en el caso concreto en cuestión el Tribunal no condenó al Fisco al pago de la multa, sino tan solo a la empresa portuaria demandada.

Ahora bien, ¿cuál es el límite del control que ejerce el TDLC? Al respecto, el Tribunal ha señalado en su sentencia $N^{\circ}$ 105, en que se discutía la legitimidad de un subsidio en el marco del Fondo de Desarrollo de las Telecomunicaciones, que:

"EL SUBSIDIO ANALIZADO EN AUTOS CONSTITUYE ENTONCES, A JUICIO DE ESTE TRIBUNAL UNA OISCRIMINACIÓN ARBITRARIA QUETIENE EFECTOS ANTICOMPETITIVOS. SIN EMBARGO, DADO QUE SU FUENTE ES LEGAL ESTE TRIBUNAL NO APLICARÁ SANCIONES RESPECTD DE LA DEMANDADA, SIN PERJUICIO DE SU FACULTAD PARA SOUITIAR LA MODIFICACIÓN O OICTACIÓN DE NORMAS, ESTABLECIDA EN EL ARTICULO $18 \mathrm{~N}^{\circ} 4$ OEL OECRETO LEY No $211 ; "$ (CONSIDERANDO 102\%).

En consecuencia, el Tribunal ha actuado en este punto con cautela. Del análisis de estos casos puede concluirse que no se considera habilitado para sancionar a los órganos de la Administración del Estado que, 
en ejercicio de potestades públicas, se aparten de lo dispuesto en el artículo $3^{\circ}$ LDC 211 , y ha reconocido un límite en los mandatos legales que cumple la Administración. A mi juicio, en esta práctica está subyacente la primacía del principio de legalidad de la actuación administrativa, aunque el Tribunal no usa esa nomenclatura ${ }^{37}$.

Entonces el TDLC se ha abstenido de aplicar multas. No obstante, ha usado frecuentemente su facultad para dictar medidas correctivas. Es lo que ocurrió en su sentencia $N^{\circ} 81$. Sin embargo, al acoger los recursos de reclamación planteados por la Administración, la Corte Suprema, por intermedio del Ministro Pierry, argumentó que:

"SÉPTIMO QUEATENTO LOANTERIOR RESULTA DE MERIDIANA CLARIDAD QUE AL ELABDRAR LAS
BASES PARA EL PRDCESO DE LICITACIÓN DE LAS FRECUENCIAS AEREAS RESTRINGIDAS MATERIA
DE ESTA CAUSA LA JUNTA DE AERONAUTICA CIVIL NO HIZO MÁS QUE DAR FIEL CUMPLIMIENTO
A LA LEGISLACIÓN VIGENTE QUE RESULTA APLICABLE EN LA ESPECIE, ES EL DECRETO SUPREMO
No 102, POR REMISIÓN DEL DECRETO LEY 2564, EL QUE ESTABLECE QUE LAS FRECUENCIAS
DEBEN SER ASIGNADAS A LA EMPRESA QUE HAYA OFRECIDO LA MAYOR CANTIDAD DE DINERD
POR CADA UNA DE ELLAS, ENCONTRÁNDDSE OBLIGADA LA JUNTA A ELABORAR LAS BASES DE
LICITACION EN CONCORDANCIA CONTALES CONDICIONES, SEGÚN EXPRESAMENTE LO DISPONE
EL INCISO SEGUNDO DEL ARTICULO 3 DE LA LEY DE AVIACIÓN:

OCTAVO: QUE DE ACUERDO A LO EXPRESADO, ENCONTRÁNDDSE VIGENTE DICHA NORMATIVA, NO LE RESULTA POSIBLE AL TRIBUNAL DE DEFENSA DE LA LIBRE COMPETENCIA ORDENAR LA MODIFICACIÓN DE LAS BASES EN LA FORMA QUE LO HIZO, DESDE QUE ELLO IMPORTA LA PDSIBILIDAD DE TENER QUE ADJUDICAR UNA O MÁS DE LAS FRECUENCIAS LICITADAS A UNA EMPRESA DISTINTA DE AQUELLA QUE OFRECIERA LA MAYOR SUMA DE DINERO, EN EL EVENTO EN QUE ESTA ÚLTIMA TUVIESE MÁS DEL 75\% DEL TOTAL DE LAS FRECUENCIAS AEREAS INTERNACIONALES DIRECTAS EXISTENTES ENTRE LA RUTA SANTIAGD-LIMA, SUMADAS LAS ASIGNADAS Y POR ASIGNAR: CONTRARIANDO CON ELLO LO QUE SE ENCUENTRA EXPRESAMENTE REGULADO POR LA LEGISLACIÓN DE AVIACIÓN COMERCIAL QUE NOS RIGE. LOS JUECES ESTÁN OBLIGADOS A CUMPLR SU FUNCIÓN APLICANDO LA LEGISLACIÓN VIGENTE, CON PRESCINDENCIA DE LA CONVICCIÓN INTIMA QUE TENGAN ACERCA DE LA BONDAD DE ESTA. DE ESTIMAR EL TRIBUNAL QUE EL REGLAMENTO DE LA LEY DE AVIACIÓN COMERCIAL, AL QUE SE REMITE EXPRESAMENTE ESTA ÚLTIMA EN MATERIA DE LICITACIÓN DE FRECUENCIAS AÉREAS INTERNACIONALES, ATENTA CONTRA LAS NORMAS QUE REGULAN LA LIBRE COMPETENCIA, EN ESTE CASO EL DECRETO LEY 211, SOLO LE ES PERMITIDO PROPDNER A LA PRESIDENTE DE LA REPÚBULCA SU MODIFICACIÓN, MÁS NO ORDENAR A LA JUNTA DE AERONÁUTICA CIVLLLLABDRAR LAS BASES CON PRESCINDENCIA DE LO QUE ÉSTE ESTABLECE, COMD ERRÓNEAMENTE LD

37 Sorprende, entonces, una sentencia como la $N^{\circ} 67$ en que se rechaza el requerimiento únicamente por razones de fondo, reconociendo el TDLC expresamente que no había una habilitación legal previa. 
HACE EN ESTA CAUSA, POR LO QUE A ESTE RESPECTD SE ACDGERÁN LAS RECLAMACIONES PLANTEADAS"38.

Es decir, frente a una ley habilitante que puede estar en conflicto con los principios de la competencia, el TDLC se inhibe de sancionar a los órganos de la Administración del Estado, pero ejerce, con amplitud, su función cooperadora, es decir, proponiendo reformas a la regulación que realicen el ideal de la competencia (artículo $18 \mathrm{~N}^{\circ} 4$ LDC). Con todo, la Corte Suprema también ha señalado los límites al ejercicio de esta facultad:

"UNDECOMO: QUE, FINALMENTE, EN CUANTD A LA DECISIÓN DEL TRIBUNAL DE EFECTUAR UNA PROPOSICIÓN A S.E. LA PRESIDENTE DE LA REPÚBLICA. CABE SEÑALAR QUE SI BIEN EL ARTICULD $18 N^{\circ} 4$ DEL DL 211 SEÑALA ENTRE LAS ATRIBUCIONES DEL TRIBUNAL DE DEFENSA DE LA LIBRE CDMPETENCIA LA DE PROPONER AL PRESIDENTE DE LA REPÚBLICA, A TRAVÉS DEL MINISTRO DE ESTADO QUE CORRESPDNDA, LA MODIFICACIÓN O DEROGACIÓN DE LOS PRECEPTOS LEGALES Y REGLAMENTARIDS QUE ESTIME CONTRARIDS A LA LIBRE COMPETENCIA, CABE SEÑALAR QUE ELLO ND LE ES PERMITIDD HACERLO EN LA PARTE RESOLUTIVA DE UNA SENTENCIA QUE SE PRONUNCIA EN UN PROCEDIMIENTO CONTENCIOSD SDBRE ACTUACIONES QUE PUEDEN CDNSTITUIR INFRACCIONES A LA LEY QUE TIENE POR OBJETO LA PROMOCIÓN Y DEFENSA DE LA LIBRE CDMPETENCIA, TODA VEZ, QUE CDMD YA SE DHO, LA COMPETENCIA EN DICHO ÁMBITD SE LA ENTREGA EL ARTICULO 26 DEL DECRETD MENCIONADD, QUE SEÑALA LAS MEDIDAS QUE ÉSTE PUEDE ADDPTAR, SIN QUE EN ELLAS SE MENCIONE TAL PRDPOSICIÓN".

Recientemente el TDLC, en la sentencia $N^{\circ} 121$ de 2012, muestra los riesgos de esta interpretación amplia de sus facultades, pues aunque reconoce el carácter discrecional de la facultad y que ha sido ejercida dentro de la habilitación legal, entra a analizar el mérito y oportunidad de la política pública, sopesándola con las alternativas que el órgano Administrativo tenía a su alcance:

"TRIGÉSIMD NDVENO: QUE SIN PERJUICIO DE LO ANTERIDR, Y A PESAR DE QUE EL DBJETNO DE PDLITICA PÚBLICA ESGRIMIDD ND ES SUSCEPTIBLE, EN SI MISMO. DE CONTROL POR PARTE DE ESTE TRIBUNALL SI CDNSTITUYE MATERIA RELEVANTE EN ESTA SEDE SI EL PROCEDIMIENTD SEGUIDO PARA ALCANZARLD PUDO HABER CAUSADD EFECTOS ANTICDMPETTINOS. LO ANTERIOR, POR CUANTO ESTE TRIBUNAL CONSIDERA QUE PUEDEN EXISTIR OTRAS FORMAS DE CDNSEGUIR LOS MISMOS OBJETIVDS DE POLITICA PÚBLICA PRODUCIENDO MENORES DISTORSIONES PARA LA LIBRE CDMPETENCIA EN EL MERCADO PREEXISTENTE DE SERVICIOS DE TRANSPDRTE DE VEHICULOS DE PASAJEROS Y DE CARGA EN LA CDNEXIÓN NIEBLA-CORRAL. ASI, DESDE EL PUNTD DE VISTA DE LA LIBRE COMPETENCIA HUBIESE SIDD PREFERIBLE, POR EJEMPLD, LICITAR LA PROVISIÓN DE SERVICIDS PORTUARIDS SEPARADAMENTE DE LA DE SERVICIDS DE TRANSPORTE Y, EN RELACIDN CON LOS SUBSIDIOS QUE SE OTORGUEN. TDDOS LDS QUUE ESTÉN EN LA MISMA SITUACIÓN DEBERIAN PODER ACCEDER A ELLOS, CDMO PDR LD DEMÁS

38 CS, 15.06.2009, rol No 1855-2009 (énfasis agregado). 
EXIGE EL ARTICULO 19 NO 22 DE NUESTRA CONSTITUCIÓN POLITICA. TAMBIÉN, PARA AFRONTAR EVENTUALES DEFICIENCIAS EN LA INFRAESTRUCTURA, HUBIESE SIDO INOCUO PARA LA LIBRE COMPETENCIA QUE LAS INVERSIONES NECESARIAS SE HUBIESEN REALIZADO CON CARGO A PRESUPUESTO PÚBLICO, O BIEN, CON CARGO A UNA TASA QUE DEBAN PAGAR TODOS LOS USUARIOS, SIN DISCRIMINACIONES DE NINGUNA ESPECIE. POR ÚLTIMO, Y TAMBIÉN A MODO DE EJEMPLO, PARA MEJORAR EVENTUALES DEFICIENCIAS DE CALIDAD O DE SEGURIDAD EN LOS SERVICIOS DE TRANSPORTE, SE PODRIA HABER INTRODUCIDO REGULACIONES DE APLICACIÓN GENERAL A LOS PROVEEDORES PARTICULARES DESTINADAS A MEJORAR LA CALIDAD DE LOS SERVICIOS, SUBSIDIANDO AL MISMO TIEMPO LAS BARCAZAS PARA TRANSPORTE DE PASAJEROS Y/O DE VEHICULOS QUE CUMPLAN DICHA REGULACIÓN

CUADRAGÉSIMO: QUEAL RESPECTO, CABETENER PRESENTE QUE,TAL COMO SE HA SEÑALADO EN OPORTUNIDADES ANTERIORES (SENTENCIA N $N^{\circ}$ 05/2010), AL IMPLEMENTAR POLITICAS PÚBLICAS O PERSEGUIR OBJETIVOS DE INTERÉS GENERAL O SECTORIAL DE DNERSA NATURALEZA, EL ESTADO DEBE INTENTAR PRODUCIR LA MINIIMA DISTORSIÓN POSIBLE EN EL FUNCIONAMIENTO COMPETTTVO DE LOS MERCADOS, POR MEDIO DEACCIONES QUE SEAN PRDPORCIONALESA LOS FINES PERSEGUIDOS. POR ENDE, LA SUJECIÓN DEL ESTADO Y SUS ORGANISMOS A LAS NORMAS DE DEFENSA DE LA LIBRE COMPETENCIA SE TRADUCE EN EL DEBER DE COMPATIBILIZAR O ARMDNIZAR LOS DIVERSOS FINES DE POLITICA PÚBLICA DE QUE SE TRATE. CON LA PROTECCIÓN DE LA LURE COMPETENCIA QUE HABRA DE RESPETAR, A LO MENDS, EN LA ELECCIÓNY DISEÑO DE LOS MECANISMOS POR MEDIO DE LOS CUALES SE PROPONGA ALCANZARLOS:"

Con todo, en este caso también elTDLC se abstuvo de adoptar medidas represivas y aunque ordenó modificar los contratos de concesión, dejó a salvo los contratos en curso ${ }^{39}$.

Recapitulando, si miramos lo hecho por el Tribunal a la luz del derecho administrativo, podemos concluir que la práctica del Tribunal es coherente

39 "Quincuagésimo séptimo: Que asimismo, y de conformidad con lo dispuesto en el artículo $3^{\circ}$ inciso primero del D.L. $N^{\circ} 211$, como medida preventiva, esto es, a fin de que la infracción sancionada precedentemente no se repita en el futuro, se ordenará al Fisco de Chile, por intermedio de los Ministerios correspondientes, después del vencimiento del Contrato, y para el evento de optar por la realización de una nueva licitación, escoger alguna de las dos siguientes alternativas: (a) licitar separadamente o adjudicar a personas diferentes y no relacionadas entre sí o con uno o más usuarios de la infraestructura portuaria, los servicios portuarios esenciales y los servicios de transporte relevantes; o bien (b) en el evento de optarse por un esquema de integración vertical entre ambos servicios, imponer al concesionario el deber de ajustar la tarifa por recalada de naves al valor que resulte de dividir el valor actualizado y anualizado de los costos y las inversiones totales en que deba incurrir y realizar el concesionario para la mantención, conservación y explotación de las obras portuarias construidas y por construir por el MOP para el atraque de embarcaciones en las rampas de Niebla y Corral, por el número total anual esperado de recaladas en el período considerado, entre todos los usuarios, incluyendo al adjudicatario de la licitación, y reduciendo del monto total de los costos e inversiones, el monto de los subsidios que se concedan para financiar tales costos e inversiones, debiendo las Bases y el Contrato respectivo especificar tal monto o establecer los elementos que sirvan para su determinación:" (Sentencia N 121) 
con la idea de que el ejercicio de potestades públicas inhibe la imposición de multas como medidas represivas, aunque no le impide hacer recomendaciones de política pública. Además, identificamos indudablemente dos sectores en que ejerce sus funciones de defensa de la competencia con toda amplitud: Estado empresario y contratación administrativa.

\section{NOTAS DE DERECHO COMPARADO}

Lo que se viene diciendo resulta totalmente coherente con la situación en el Derecho Comparado, en que se llega a las mismas conclusiones que se describen en este artículo. Nos referiremos brevemente a la situación en España y Estados Unidos. Es necesario advertir que en ambos ordenamientos se analizan conjuntamente, tanto la aplicación del derecho de la competencia a los órganos administrativos como la actuación privada en ejercicio de títulos habilitantes creados por la administración.

\section{España}

En el derecho español, si bien se afirma que la mera presencia de la Administración no puede ser cobertura de una conducta contraria a las prohibiciones del derecho de la competencia, obviamente la Administración puede incidir en el mercado en el ejercicio de potestades.

"CUANDO EL CONFLICTOVERSA SOBRE ESTE EJERCICIO, LAS AUTORIDADES DE COMPETENCIA NO DETERMINAN LA EXISTENCIA DE INFRACCIÓN Y ADEMAS SON CUESTIONES RESERVADAS A LA JURISDICCIÓN CDNTENCIOSO-ADMINISTRATIVA"40.

Se han considerado que son intervenciones en el mercado en ejercicio de potestades públicas y, por lo tanto, exentas de control de acuerdo con las normas de la libre competencia las siguientes conductas: aprobación de planes urbanísticos, concesión de licencias para la venta ambulante, concesión de licencias para máquinas expendedoras, concesión de autorizaciones para la ocupación temporal del dominio público, concesión de uso de atraques en puerto, gestión en el ámbito portuario mediante convenio administrativo y la gestión patrimonial por parte de un organismo autónomo.

40 CASES, Lluis. "Regulación y competencia. Límites y conexiones" en MUÑOZ MACHADO, Santiago y ESTEVE PARDO, José (eds.) Derecho de la Regulación Económica. I. Fundamentos e instituciones de la regulación. Madrid, lustel, 2009, p. 452. 
El Tribunal español de Defensa de la Competencia afirmó, en sentencia de 20 de marzo de 1998, que, al otorgarse una licencia, un municipio:

"ESTÁ EN CONSECUENCIA ACTUANDO COMO REGULADORYNO CDMO DPERADDR ECONOMICD Y LA ACTMIDAD QUE DESARROLLA ES UNA ACTMIDAD REGULATORIA QUE NO PUEDE SER ENJUICADA DE ACUERDO CON LAS NORMAS DE LA COMPETENCIA"4!

En cambio, si la Administración actúa como operador económico le resultan plenamente aplicables las prohibiciones del derecho de la competencia. La distinción entre un acto realizado en cuanto regulador y otro en cuanto operador económico es crucial. Así, se han considerado como actos realizados en cuanto regulador (y no como operador): la constitución de una empresa de gestión de suelo y la decisión sobre ejercer (o no) una actividad económica. En cambio, el posterior ejercicio de la actividad elegida, está plenamente sujeto al derecho de la competencia ${ }^{42}$.

\section{Estados Unidos}

Tal como reconoce Nehme Zalaquett, en Estados Unidos la ley ha fijado excepciones al derecho de la competencia y la Corte Suprema ha sido muy deferente hacia la regulación mediante las agencias administrativas ${ }^{43}$.

La tensión subyacente es que la actuación estatal puede ser más dañina que la acción privada y, en cuanto constituye una regulación pública, no puede ser superada mediante una competencia más agresiva por parte de las empresas. Sin embargo, un Estado democrático debe tener espacios para promover el interés público y las personas deben tener la libertad para influir en esas decisiones.

En los Estados Unidos resulta perfectamente posible que el derecho federal exceptúe la aplicación del derecho de la competencia respecto de conductas privadas, sea explícita o implícitamente. Esto último, cuando resulta necesaria para que funcione un régimen regulatorio ${ }^{44} \mathrm{o}$

41 Ibíd. pp. 452-455.

42 lbíd. pp. 453-454, 460-46I.

43 NEHME ZALAQUETT, Nicole, Op. cit., p. 355.

44 United States v. National Association of Securities Dealers, (1975); Gordon v. New York Stock Exchange, (1975).Vid. FOX, Eleanor, "The framework for U.S. antitrust law. Enforcement, procedure and the scope of the law", en Cases and Materiols on United Stotes Antitrust in Global Context, $3^{\mathrm{a}}$ ed., St. Paul MN, West Publishing, 2012, Borrador sin editar. 
bien cuando hay contradicción total entre el derecho de la competencia y la ley sectorial ${ }^{45}$. El conflicto entre la regulación federal y el derecho de la competencia se resuelve simplemente recurriendo al principio de especialidad 46 .

Ahora bien, en cuanto a la aplicación del derecho de la competencia a entidades de naturaleza pública, el caso líder es Parker v. Brown (1943), en que un productor de pasas en California impugnó un programa estadual que creaba -siguiendo los lineamientos del New Deal- un régimen centralizado de comercialización para todos los productores. La Corte resolvió que la Sherman Act no era aplicable, pues esta prohibe restricciones a la competencia originadas por privados, no por los estados ${ }^{47}$.

Con posterioridad, los casos que se presentaron fueron distintos. Los demandados usaron la doctrina de Parker como defensa. Es decir, amparándose en alguna regulación estadual se pidió el rechazo de las demandas basadas en el derecho de la competencia. Hoy en día, se entiende que esta defensa sólo puede usarse (i) si el estado ha ordenado expresamente la conducta o si una regulación tiene la intención expresa de desplazar la competencia, (iii) si el estado supervisa activamente la regulación, y (iii) siempre que la regulación Federal no disponga lo contrario (California Retail Liquor Dealers Association v. Midcal Aluminum $(0 .-1980)^{48}$.

El desarrollo de estas sentencias y precedentes ha llevado a la doctrina a concluir:

45 Credit Suisse v. Billing (2007). Ibíd.

46 EI TDLC se ha inclinado a favor de esta idea en su sentencia $N^{\circ}$ 121: "Quincuagésimo segundo: Que, en razón de lo anterior, no es posible considerar la conformidad con la normativa especial que regula una actividad -en este caso, la de concesiones de obra pública-como eximente de responsabilidad por el incumplimiento de la normativa general de defensa de la libre competencia, especialmente si no se verifica en la especie una situación de contradicción entre ambas que impida cumplirlas simultáneamente y, por el contrario, existe un ámbito de discrecionalidad en la conducta de Somarco constitutiva de infracción, toda vez que bien pudo fijar, por debajo del máximo autorizado por su contrato, cualquier tarifa que no produjera o tendiera a producir los efectos contrarios a la libre competencia antes descritos;"

47 Hovenkamp, Herbert, "Antitrust. Black Letter Outlines", 5 ed., St. Paul MN, West Publishing, 2011, p. 345.

48 Ibíd., p. 346. 
"EN BREVE, AQUELLA ACCIÓN QUE ES UN ACTO DEL ESTADO MISMO NO ESTÁ SUJETA AL DERECHO DE LA COMPETENCIA. UNA ACTUACIÓN DE UN MUNICIPIO, AMPARADA EN UNA POLITICA CLARAMENTE ESTABLECIDA POR EL ESTADO DE REEMPLAZAR LA COMPETENCIA POR LA REGULACIÓN O QUE ESTANDO AUTORIZADA POR EL ESTADO IMPLIQUE UNA RESTRICCIÓN PREVISIBLEMENTE ANTICOMPEIITVA, TAMBIÉN ESTÁ EXENTA. UN ACTO DE UN PRIVADO QUE ESTÁ ORDENADO O PERMITIDO POR EL ESTADO (O POR UN GOBIERNO LOCAL DEBIDAMENTE AUTORIZADO) EN EJECUCIÓN DE UNA POLITICA CLARAMENTE ESTABLECIDA Y QUE ESTÁ ACTVAMENTE SUPERVISADO POR EL GOBIERNO ESTADUAL O LOCAL ESTÁ EXENTO. SI ELALCANCE DE ESTAS EXENCIONES PARECE DEMASIADD AMPLIO, EL FORO ADECUADO ES EL CONGRESO"49.

\section{CONCLUSIONES}

La LDC 21 I dota al Tribunal de Defensa de la Libre Competencia con amplias facultades para aplicar multas a todo sujeto que intervenga en conductas anticompetitivas.

Tratándose de los órganos del Estado, la potestad del TDLC resulta problemática. Aunque el Tribunal no se ha cuestionado el fundamento de su competencia, hay buenas razones para ser cauteloso.

El principio de legalidad debiera amparar toda actuación de los órganos de la Administración del Estado en este ámbito.

Pero es un hecho indiscutible que el TDLC se ha pronunciado en numerosas ocasiones cuestionando la actuación administrativa.

Sin embargo, esta amplitud debe ser mirada con cautela. En la práctica, el TDLC ha sido deferente con la Administración, absteniéndose de adoptar medidas represivas. Así, cuando ha acogido acciones en contra de órganos de la Administración que ejercen potestades, amparadas por la presunción de legalidad, se ha abstenido de aplicar multas, pero ha impartido órdenes para que corrijan sus actos desde el punto de vista de la competencia.

49 Traducción libre de "In short, action that is an act of the state itself is not covered by the antitrust laws. An act of a city that is authorized by the state pursuant to a clearly articulated policy to replace competition with regulation or that is authorized by the state and entails restraints that are foreseeably anticompetitive is exempt. An act of a private party that is directed or permitted by the state (or by an authorized local government) pursuant to such a clearly articulated policy and that is actively supervised by state or local government is exempt. If the scope of these resulting exemptions is unduly broad, recourse lies with Congress". FOX, Eleanor, Op. cit. 
En definitiva, la amplitud con que el Tribunal de Defensa de la Competencia ha entendido su competencia hasta hoy debe ser moderada a la luz del sistema de derecho público y las exigencias de un Estado democrático. 


\section{BIBLIOGRAFÍA}

BREYER, Stephen; STEWART, Richard B.; SUNSTEIN, Cass; VERMEULE, Adrian y HERZ, Michael E., "Administrative Law and Regulatory Policy", $7^{\mathrm{a}}$ ed., New York, Wolters Klower, 2011.

CASES, Lluis. "Reguiación y competencia. Límites y conexiones" en Muñoz Machado, Santiago (ed.) Derecho de la Regulación Económica. I. Fundamentos e instituciones de la regulación. Madrid, lustel, 2009.

FERMANDOIS VÖHRINGER, Arturo, "Derecho Constitucional Económico", T. I, $2^{\text {a }}$ ed.، Santiago, Ed. Universidad Católica, 2006.

FERRADA BÓRQUEZ, Juan Carlos.' 'Las potestades y privilegios de la Administración pública en el régimen administrativo chileno", en Revista de Derecho (Valdivia) [online]. 2007, vol.20, n.2, pp. 69-94.

FOX, Eleanor,"The framework for U.S. antitrust law. Enforcement, procedure and the scope of the law", en Coses and Materials on United States Antitrust in Global Context, $3^{\text {a }}$ ed., St. Paul MN,West Publishing, 20 I 2, borrador sin editar.

GARCÍA DE ENTERRÍA, Eduardo y FERNÁNDEZ, Tomás-Ramón, "Curso de Derecho Administrativo", T. I، $12^{\text {a }}$ ed. Madrid-Buenos Aires, ThompsonCivitas-La Ley, 2004.

GARCÍA VITORIA, Ignacio, "La libertad de empresa: ¿Un terrible derecho?", Madrid, Centro de Estudios Políticos y Constitucionales, 2008.

HOVENKAMP, Herbert, "Antitrust. Black Letter Outlines", $5^{\text {a }}$ ed., St. Paul MN. West Publishing, 2011.

HUERGO LORA, Alejandro, "Las sanciones administrativas", Madrid, lustel, 2007.

MAURER, Hartmut، "Derecho Administrativo. Parte General', Madrid, Marcial Pons، 2011.

MORAGA KLENNER, Claudio, "Contratación administrativa", Santiago, Ed. Jurídica de Chile, 2007.

NEHME ZALAQUETT، Nicole, "Aplicación de las normas de defensa de la competencia a los órganos de la Administración del Estado", en Tribunal de Defensa de la Libre Competencia (ed.) La Libre Competencia en el Chile del Bicentenario, Santiago, Thomson Reuters, 2010.

PANTOJA BAUZÁ, Rolando, "La organización administrativa del Estado", Santiago, Ed. Jurídica de Chile, 2004.

SAAVEDRA FERNÁNDEZ, Rubén. "Discrecionalidad Administrativa". Santiago, Abeledo Perrot, 20II. 
SANTAMARÍA PASTOR, Juan Alfonso, "Principios Generales de Derecho Administrativo", T. I, Madrid, lustel, 2006.

VALDÉS PRIETO, Domingo, "Libre Competencia y Monopolio", Santiago, Ed. Jurídica de Chile, 2006. 
ANEXO

\begin{tabular}{|c|c|c|c|c|c|}
\hline Rol & Sentencia & $\begin{array}{c}\text { Actuación } \\
\text { administrativa }\end{array}$ & Órgano administrativo & Medida adoptada & Resultado del recurso \\
\hline$C-209-10$ & 123 & $\begin{array}{l}\text { Actividad presta- } \\
\text { cional del Estado }\end{array}$ & $\begin{array}{l}\text { Dirección de Bienestar Social } \\
\text { de la Armada }\end{array}$ & Rechaza & \\
\hline$C-219-11$ & 121 & Concesión & $\begin{array}{l}\text { Ministerio de Obras Públicas } \\
\text { y Ministerio de Transportes y } \\
\text { Telecomunicaciones }\end{array}$ & $\begin{array}{l}\text { Acoge. No aplica multa. } \\
\text { Ordena modificar con- } \\
\text { tratos en el futuro (no } \\
\text { los presentes) } \\
\end{array}$ & \\
\hline$C-206-10$ & 114 & Licitación & $\begin{array}{l}\text { Dirección de Compras y } \\
\text { Contratación Pública }\end{array}$ & Rechaza & \\
\hline$C-179-08$ & 105 & Subvención & $\begin{array}{l}\text { Ministerio de Transportes y } \\
\text { Telecomunicaciones }\end{array}$ & $\begin{array}{l}\text { Acoge. No aplica multa. } \\
\text { Ordena adoptar medi- } \\
\text { das correctivas }\end{array}$ & - \\
\hline$C-127.07$ & 100 & Concesión & Fisco & $\begin{array}{l}\text { Acoge. No aplica } \\
\text { medida respecto del } \\
\text { Fisco }\end{array}$ & $\begin{array}{l}\mathrm{CS} \text {, rol } \mathrm{N}^{\circ} 6100-2010 . \\
\text { Revoca }\end{array}$ \\
\hline$C-172-08$ & 92 & Licitación & Municipalidad de Antofagasta & Rechaza & - \\
\hline C-189-09 & 91 & Licitación & Municipalidad de Providencia & Rechaza & - \\
\hline$C-171-08$ & 89 & Licitación & Ministerio de Hacienda & Rechaza & $\begin{array}{l}\mathrm{CS} \text {, rol } \mathrm{N}^{\circ} 9148-2009 . \\
\text { Confirma }\end{array}$ \\
\hline$C-148-07$ & 81 & Licitación & Junta de Aeronáutica Civil & $\begin{array}{l}\text { Acoge. No aplica multa. } \\
\text { Ordena adoptar medi- } \\
\text { das correctivas }\end{array}$ & $\begin{array}{l}\text { CS, rol No } 1855-2009 . \\
\text { Revoca }\end{array}$ \\
\hline
\end{tabular}




\begin{tabular}{|c|c|c|c|c|c|}
\hline Rol & Sentencia & $\begin{array}{c}\text { Actuación } \\
\text { administrativa }\end{array}$ & Órgano administrativo & Medida adoptada & Resultado del recurso \\
\hline C-137-07 & 77 & Licitación & Municipalidad de Curicó & Acoge. Aplica multa & $\begin{array}{l}\text { CS, rol No } 7796-2008 . \\
\text { Confirma }\end{array}$ \\
\hline$C-124-07$ & 67 & $\begin{array}{l}\text { Actividad empre- } \\
\text { sarial del Estado }\end{array}$ & $\begin{array}{l}\text { CONAF y Ministerio de } \\
\text { Agricultura }\end{array}$ & Rechaza & - \\
\hline C-70-05 & 44 & Licitación & $\begin{array}{l}\text { Ministerio de Transportes y } \\
\text { Telecomunicaciones y junta } \\
\text { de Aeronáutica Civil }\end{array}$ & $\begin{array}{l}\text { Acoge. No aplica multa. } \\
\text { Ordena adoptar medi- } \\
\text { das correctivas }\end{array}$ & - \\
\hline C-45-04 & 37 & Licitación & $\begin{array}{l}\text { Municipalidad de San } \\
\text { Bernardo }\end{array}$ & Acoge. No aplica multa & $\begin{array}{l}\text { CS, rol No 2657-2006. } \\
\text { Confirma }\end{array}$ \\
\hline C-72-05 & 34 & Licitación & Municipalidad de Cauquenes & $\begin{array}{l}\text { Acoge. No aplica multa. } \\
\text { Ordena adoptar medi- } \\
\text { das correctivas }\end{array}$ & - \\
\hline$C-21-04$ & 20 & Licitación & Municipalidad de Santiago & $\begin{array}{l}\text { Rechaza. Ordena adop- } \\
\text { tar medidas correctivas }\end{array}$ & - \\
\hline$C-37-0^{\prime} 4$ & 14 & Licitación & $\begin{array}{l}\text { Ministerio de Transportes y } \\
\text { Telecomunicaciones }\end{array}$ & Rechaza & CS. Confirma \\
\hline$C-54-04$ & 13 & Licitación & $\begin{array}{ll}\text { Subsecretaría de } \\
\text { Telecomunicaciones }\end{array}$ & Rechaza & - \\
\hline$C-29-04$ & 11 & Licitación & $\begin{array}{l}\text { Ministerio de Transportes y } \\
\text { Telecomunicaciones }\end{array}$ & Rechaza & - \\
\hline$C-32-04$ & 4 & Licitación & $\begin{array}{l}\text { Municipalidad de Puente } \\
\text { Alto }\end{array}$ & "Aprueba" & - \\
\hline
\end{tabular}

